PROCEEDINGS OF THE

AMERICAN MATHEMATICAL SOCIETY

Volume 140, Number 6, June 2012, Pages 2117-2126

S 0002-9939(2011)11205-X

Article electronically published on October 21, 2011

\title{
SOLITON SOLUTIONS OF THE MEAN CURVATURE FLOW AND MINIMAL HYPERSURFACES
}

\author{
NORBERT HUNGERBÜHLER AND THOMAS METTLER
}

(Communicated by Jianguo Cao)

\begin{abstract}
Let $(M, g)$ be an oriented Riemannian manifold of dimension at least 3 and $\mathbf{X} \in \mathfrak{X}(M)$ a vector field. We show that the Monge-Ampère differential system (M.A.S.) for $\mathbf{X}$-pseudosoliton hypersurfaces on $(M, g)$ is equivalent to the minimal hypersurface M.A.S. on $(M, \bar{g})$ for some Riemannian metric $\bar{g}$ if and only if $\mathbf{X}$ is the gradient of a function $u$, in which case $\bar{g}=$ $e^{-2 u} \mathrm{~g}$. Counterexamples to this equivalence for surfaces are also given.
\end{abstract}

\section{INTRODUCTION}

Recall that a smooth family of hypersurfaces $F_{t}: \Sigma^{n} \rightarrow M^{n+1}, t \geq 0$, in a Riemannian manifold $(M, g)$ is called a solution of the mean curvature flow (M.C.F.) on $(0, T), T>0$, if

$$
\begin{aligned}
\frac{d}{d t} F_{t} & =-\mathbf{H}, & & \text { on } \Sigma \times(0, T), \\
F_{0} & =f, & & \text { on } \Sigma,
\end{aligned}
$$

where $f: \Sigma \rightarrow M$ is a given initial hypersurface and $\mathbf{H}$ denotes the mean curvature vector of $F_{t}(\Sigma)$. Suppose there exists a conformal Killing vector field $\mathbf{X}$ on $M$ with flow $\varphi: M \times \mathbb{R} \rightarrow M$. A family of hypersurfaces $F_{t}$ is said to be a soliton solution of the M.C.F. with respect to the conformal Killing vector field $\mathbf{X}$ if $\tilde{F}_{t}=\varphi^{-1}\left(F_{t}, t\right)$ is stationary in the normal direction, i.e. $\tilde{F}_{t}(\Sigma)$ is the fixed hypersurface $f(\Sigma)$. In 8 it was shown that for a given initial hypersurface $f: \Sigma \rightarrow M$ to give rise to a soliton solution of the mean curvature flow it is necessary that

$$
\mathbf{H}+\mathbf{X}^{\perp}=0,
$$

where $\perp$ denotes the $g$-orthogonal projection onto the normal bundle of the hypersurface $f: \Sigma \rightarrow M$. If $\mathbf{X}$ is Killing, then (1.1) is also sufficient.

Soliton solutions have played an important rôle in the development of the theory of the M.C.F. Such solutions served, e.g., as tailor-made comparison solutions to investigate the development of singularities (e.g. Angenent's self-similarly shrinking doughnut; see [3]). Actually, soliton solutions appear as blowups of so-called type II

Received by the editors February 11, 2011.

2010 Mathematics Subject Classification. Primary 49Q05.

Key words and phrases. Mean curvature flow, soliton solutions, minimal hypersurfaces, MongeAmpère systems, equivalence problem.

Research for this article was carried out while the authors were supported by the Swiss National Science Foundation, the first author by the grant 200020-124668, and the second by the postdoctoral fellowship PBFRP2-133545.

(C)2011 American Mathematical Society Reverts to public domain 28 years from publication 
singularities of the flow of plane curves (see [2]). Moreover, soliton solutions turn out to enjoy certain stability properties and allow some insight into the behaviour of the mean curvature flow viewed as a dynamical system (see [8, [13] and [6]).

In 8 the boundary value problem for rotating soliton solutions has been discussed. The corresponding local existence result has been generalised to arbitrary Killing fields in [9]. For rotating solitons in the Euclidean plane, so-called yin-yang curves, a quantity was identified that remains invariant along the curve (see 9]). This invariant made it possible to show that yin-yang curves share fundamental geometric properties with geodesic curves. In 9] the corresponding results have been generalised to arbitrary soliton curves on surfaces (see Figure 1). In addition,

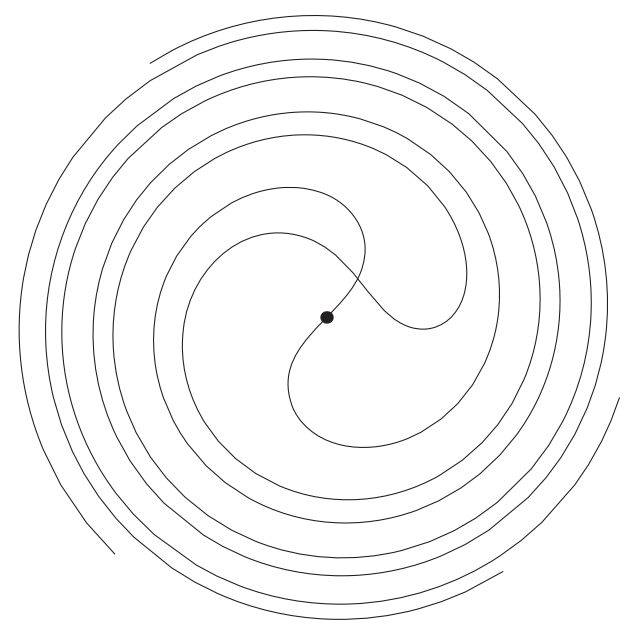

Figure 1. If the Gaussian curvature of the simply connected ambient surface is less than or equal to 0 , then two soliton curves intersect in at most one point. This fact is illustrated here by two yin-yang curves rotating about the origin.

it was observed in 9 that translating solitons in the Euclidean plane, the so-called grim reaper curves, actually are geodesics with respect to a conformally deformed Riemannian metric. Therefore the natural question arose whether soliton curves are (at least locally) always geodesic curves with respect to a modified Riemannian metric. This is not the case. On a surface $(M, g)$, the solutions of (1.1) are immersed curves on $M$ which may be reparametrised to become geodesics of the Weyl connection $\nabla_{g, \mathbf{X}}$ given by

$$
\left(\mathbf{Y}_{1}, \mathbf{Y}_{2}\right) \mapsto\left(D_{g}\right)_{\mathbf{Y}_{1}} \mathbf{Y}_{2}-g\left(\mathbf{Y}_{1}, \mathbf{Y}_{2}\right) \mathbf{X}+g\left(\mathbf{X}, \mathbf{Y}_{1}\right) \mathbf{Y}_{2}+g\left(\mathbf{X}, \mathbf{Y}_{2}\right) \mathbf{Y}_{1}
$$

where we have written $D_{g}$ for the Levi-Civita connection of $g$. The equation (1.1) is parametrisation invariant, and thus its solutions are naturally interpreted as the geodesics of a projective structure on $M$. Recall that a projective structure is an equivalence class of affine torsion-free connections, where two such connections are said to be equivalent if they have the same geodesics up to parametrisation. 
Recently in 4, Bryant, Dunajski and Eastwood determined the necessary and sufficient local conditions for an affine torsion-free connection to be projectively equivalent to a Levi-Civita connection. Applying their result:1 it follows that the Weyl connection whose geodesics are the yin-yang curves is not projectively equivalent to a Levi-Civita connection. However Jürgen Moser conjectured 2 that soliton curves can at least locally be interpreted as geodesics of a Finsler metric. Recent results about Finsler metrisability of path geometries by Álvarez-Paiva and Berck 1 show that this is indeed the case. Of course, one can ask analogous questions also for higher-dimensional solitons. Before we do that, we generalise the notion of soliton solutions slightly.

Definition. A hypersurface $f: \Sigma \rightarrow M$ solving (1.1) for some vector field $\mathbf{X} \in$ $\mathfrak{X}(M)$ will be called an $\mathbf{X}$-pseudosoliton hypersurface of $(M, g)$.

Note that the $\mathbf{0}$-pseudosoliton hypersurfaces are the minimal hypersurfaces of $(M, g)$. It was observed in [13] (see also [7]) that solitons with respect to gradient vector fields correspond to minimal hypersurfaces. However it was left open if such a correspondence holds when the vector field is not the gradient of a smooth function. In this short article we provide an answer using the framework of Monge-Ampère differential systems.

In $\S 2$ we will associate to the $\mathbf{X}$-pseudosoliton hypersurface equation on $(M, g)$ a Monge-Ampère system (M.A.S.) on the unit tangent bundle of $M$ whose Legendre integral manifolds, which satisfy a natural transversality condition, locally correspond to $\mathbf{X}$-pseudosoliton hypersurfaces on $M$. We then show that for a gradient vector field $\mathbf{X}=\nabla_{g} u$ on $M$, the $\mathbf{X}$-pseudosoliton M.A.S. is equivalent to the minimal hypersurface M.A.S. on $\left(M, e^{-2 u} g\right)$. This was already shown in 13, albeit expressed in different language. We complete the picture by proving the

Theorem 2.3. The $\mathbf{X}$-pseudosoliton M.A.S. on an oriented Riemannian manifold $(M, g)$ of dimension $n+1 \geq 3$ is equivalent to a minimal hypersurface M.A.S. if and only if $\mathbf{X}$ is a gradient vector field.

Theorem 2.3 is wrong for $n=1$, i.e. the case of curves on surfaces. We provide counterexamples and comment on the necessary and sufficient conditions for $\mathbf{X}$ in the surface case. Theorem 2.3 provides an answer to the equivalence problem for specific M.A.S. in arbitrary dimension $n+1 \geq 3$. The equivalence problem for general M.A.S. has been studied for 5 -dimensional contact manifolds in [5] and in various low dimensions in [11.

Throughout the article all manifolds are assumed to be connected, and smoothness, i.e. infinite differentiability, is assumed.

\section{Equivalence of The SOLITON AND MINIMAL HYPERSURFACE EQUATION}

2.1. Monge-Ampère systems. Let $N$ be a $(2 n+1)$-dimensional manifold equipped with a contact structure, meaning a maximally nonintegrable codimension 1 subbundle $D \subset T N$ which we assume to be given by the kernel of a globally

\footnotetext{
${ }^{1}$ Since the computations are somewhat complex, they have been carried out using Maple. The Maple file can be obtained from the authors upon request.

${ }^{2}$ Stated on the occasion of a seminar talk of the first author at the Institute for Mathematical Research (FIM) at ETH Zürich, March 1999.
} 
defined contact form $\theta$. Recall that an $n$-dimensional submanifold $f: \Sigma \rightarrow N$ which satisfies $f^{*} \theta=0$ is called a Legendre submanifold of $(N, D)$. A Monge-Ampère differential system on $(N, D)$ is a differential ideal $\mathcal{M} \subset \mathcal{A}^{*}(N)$ in the exterior algebra of differential forms on $N$ given by

$$
\mathcal{M}=\{\theta, \mathrm{d} \theta, \varphi\}
$$

where $\varphi \in \mathcal{A}^{n}(N)$ is an $n$-form 3 The brackets \{\} denote the algebraic span of the elements within; i.e., the elements of $\mathcal{M}$ may be written as

$$
\alpha \wedge \theta+\beta \wedge \mathrm{d} \theta+\gamma \wedge \varphi
$$

where $\alpha, \beta, \gamma$ are differential forms on $N$. Note that $\mathcal{M}$ is indeed a differential ideal since $\mathrm{d} \varphi$ lies in the contact ideal $\mathcal{C}=\{\theta, \mathrm{d} \theta\}$; cf. [5]. A Legendre submanifold of $(N, D)$ which pulls back to 0 the $n$-form $\varphi$ as well will be called a Legendre integral manifold of $\mathcal{M}$. Two Monge-Ampère systems $(N, \mathcal{M})$ and $(\bar{N}, \overline{\mathcal{M}})$ are called equivalent if there exists a diffeomorphism $\psi: N \rightarrow \bar{N}$ identifying the two ideals. Note that this implies that $\psi$ is a contact diffeomorphism.

2.2. Minimal hypersurfaces via frames. In order to fix notation we review the description of minimal hypersurfaces using moving frames. For $n \geq 1$, let $(M, g)$ be an oriented Riemannian $(n+1)$-manifold, $\pi: F \rightarrow M$ its right principal $S O(n+1)$ bundle of positively oriented orthonormal frames and $\tau: U \rightarrow M$ its (sphere) bundle of unit tangent vectors. Write the elements of $F$ as $\left(p, e_{0}, \ldots, e_{n}\right)$, where $p \in M$ and $e_{0}, \ldots, e_{n}$ is a positively oriented $g$-orthonormal basis of $T_{p} M$. The Lie group $S O(n+1)$ acts smoothly from the right by

$$
\left(p, e_{0}, \ldots, e_{n}\right) \cdot r=\left(p, \sum_{i=0}^{n} e_{i} r_{i 0}, \ldots, \sum_{i=0}^{n} e_{i} r_{i n}\right),
$$

where $r_{i k}$ for $i, k=0, \ldots, n$ denote the entries of the matrix $r$. The map $\nu: F \rightarrow U$, given by $\left(p, e_{0}, \ldots, e_{n}\right) \mapsto\left(p, e_{0}\right)$, is a smooth surjection whose fibres are the $S O(n)$ orbits and thus makes $F$ together with its right action into an $S O(n)$-bundle over $U$. Here we embed $S O(n)$ as the Lie subgroup of $S O(n+1)$ given by

$$
\left\{\left(\begin{array}{ll}
1 & 0 \\
0 & r
\end{array}\right) \in S O(n+1), r \in S O(n)\right\} .
$$

Let $\omega_{i} \in \mathcal{A}^{1}(F)$ denote the tautological forms of $F$ given by

$$
\left(\omega_{i}\right)_{\left(p, e_{0}, \ldots, e_{n}\right)}(\xi)=g_{p}\left(e_{i}, \pi^{\prime}(\xi)\right),
$$

and $\omega_{i k} \in \mathcal{A}^{1}(F)$ the Levi-Civita connection forms which satisfy $\omega_{i k}+\omega_{k i}=0$. The dual vector fields to the coframing $\left(\omega_{i}, \omega_{i k}\right), i<k$, will be denoted by $\left(\mathbf{W}_{i}, \mathbf{W}_{i k}\right)$. Recall that we have the structure equations

$$
\begin{gathered}
\mathrm{d} \omega_{i}+\sum_{k=0}^{n} \omega_{i k} \wedge \omega_{k}=0, \\
\mathrm{~d} \omega_{i k}+\sum_{l=0}^{n} \omega_{i l} \wedge \omega_{l k}=\Omega_{i k},
\end{gathered}
$$

\footnotetext{
${ }^{3}$ More generally one can define an M.A.S. to be a differential ideal which is only locally generated by a contact ideal and an $n$-form. However for our purposes the above definition is sufficient.
} 
where $\Omega_{i k} \in \mathcal{A}^{2}(F)$ are the curvature forms. Denote by $\hat{\omega}_{i}$ the wedge product of the forms $\omega_{1}, \ldots, \omega_{n}$, with the $i$-th form omitted:

$$
\hat{\omega}_{i}=\omega_{1} \wedge \cdots \wedge \omega_{i-1} \wedge \omega_{i+1} \wedge \cdots \wedge \omega_{n} .
$$

For $n=1$ set $\hat{\omega}_{1} \equiv 1$. Note that the forms

$$
\begin{aligned}
\theta & =\omega_{0}, \\
\omega & =\omega_{1} \wedge \cdots \wedge \omega_{n}, \\
\mu & =-\frac{1}{n} \sum_{i=1}^{n}(-1)^{i-1} \omega_{0 i} \wedge \hat{\omega}_{i}
\end{aligned}
$$

are $\nu$-basic, i.e. pullbacks of forms on $U$ which, by abuse of language, will also be denoted by $\theta, \omega, \mu$. Since

$$
\mathrm{d} \omega_{0}=-\sum_{k=1}^{n} \omega_{0 k} \wedge \omega_{k}
$$

the 1 -form $\theta$ is a contact form. Note also that

$$
\mathrm{d} \omega+(-1)^{n-1} n \mu \wedge \theta=0 .
$$

The geometric significance of these forms is the following. Suppose $f: \Sigma \rightarrow M$ is an oriented hypersurface and $\mathcal{G}_{f}: \Sigma \rightarrow U$ its orientation-compatible Gauss lift. In other words the value of $\mathcal{G}_{f}$ at $p \in \Sigma$ is the unique unit vector at $f(p)$ which is $g$ orthogonal to $f^{\prime}\left(T_{p} \Sigma\right)$ and together with a positively oriented basis of $T_{p} \Sigma$ induces the positive orientation of $T_{f(p)} M$. By construction we have

$$
\mathcal{G}_{f}^{*} \theta=0
$$

and simple computations show that

$$
\mathcal{G}_{f}^{*} \omega=\omega_{f^{*} g},
$$

where $\omega_{f^{*} g}$ denotes the Riemannian volume form on $\Sigma$ induced by $f^{*} g$. Suppose $\tilde{f}: V \subset \Sigma \rightarrow F$ is a local framing covering $\mathcal{G}_{f}$ and $f$. Then pulling back (2.4) and using (2.2) gives

$$
\sum_{k=1}^{n} \tilde{f}^{*} \omega_{0 k} \wedge \tilde{f}^{*} \omega_{k}=0
$$

The independence (2.5) implies that the forms $\varepsilon_{i}=\tilde{f}^{*} \omega_{i}$ are linearly independent, and thus Cartan's lemma yields the existence of functions $h_{i k}: V \rightarrow \mathbb{R}$, symmetric in the indices $i, k$, such that

$$
\tilde{f}^{*} \omega_{0 i}=\sum_{k=1}^{n} h_{i k} \varepsilon_{k}
$$

In particular we have

$$
\mathcal{G}_{f}^{*} \mu=-H \varepsilon_{1} \wedge \cdots \wedge \varepsilon_{n},
$$

where $H=\frac{1}{n} \sum_{i=1}^{n} h_{i i}$ is the mean curvature of the hypersurface $f: \Sigma \rightarrow M$. Conversely if $\mathcal{G}: N \rightarrow U$ is an orientable $n$-submanifold with $\mathcal{G}^{*} \theta=0$ and $\mathcal{G}^{*} \omega \neq 0$, then $\tau \circ \mathcal{G}: N \rightarrow M$ is an immersion. Shrinking $N$ if necessary we can assume that $f=\tau \circ \mathcal{G}: N \rightarrow M$ is a hypersurface, which can be oriented in such a way that its 
Gauss lift agrees with $\mathcal{G}$. Thus the Legendre integral manifolds $\mathcal{G}: \Sigma \rightarrow U$ of the M.A.S. $\mathcal{M}_{g}$ on $U$, given by

$$
\mathcal{M}_{g}=\{\theta, \mathrm{d} \theta, \mu\},
$$

which satisfy the transversality conditions $\mathcal{G}^{*} \omega \neq 0$, locally correspond to minimal hypersurfaces on $(M, g)$.

2.3. $\mathbf{X}$-pseudosoliton hypersurfaces via frames. Given a vector field $\mathbf{X}$ on $M$, define the functions $X_{i}: F \rightarrow \mathbb{R}$ by

$$
\left(p, e_{0}, \ldots, e_{n}\right) \mapsto g_{p}\left(\mathbf{X}(p), e_{i}\right) .
$$

Of course $X_{0}$ is the $\nu$-pullback of a function on $U$, which will be denoted by $X$. Using (1.1) and (2.6) it follows that an oriented hypersurface $f: \Sigma \rightarrow M$ is an $\mathrm{X}$-pseudosoliton hypersurface if and only if

$$
\mathcal{G}_{f}^{*}(\mu-X \omega)=0 .
$$

Thus the Legendre integral manifolds $\mathcal{G}: \Sigma \rightarrow U$ of the M.A.S. $\mathcal{M}_{g, \mathbf{X}}$ on $U$, given by

$$
\mathcal{M}_{g, \mathbf{X}}=\{\theta, \mathrm{d} \theta, \mu-X \omega\},
$$

which satisfy the transversality conditions $\mathcal{G}^{*} \omega \neq 0$, locally correspond to $\mathbf{X}$-pseudosoliton hypersurfaces on $(M, g)$. Now suppose $\mathbf{X}$ is a gradient vector field $\mathbf{X}=\nabla_{g} u$ for some smooth function $u: M \rightarrow \mathbb{R}$. Let $\bar{g}=e^{-2 u} g, \bar{\pi}: \bar{F} \rightarrow M$ denote the bundle of positively oriented $\bar{g}$-orthonormal frames with canonical coframing $\bar{\omega}_{i}, \bar{\omega}_{i k}$ and $\tilde{\psi}: F \rightarrow \bar{F}$ the map which scales a $\bar{g}$-orthonormal frame by $e^{u}$. Then by definition

$$
\tilde{\psi}^{*} \bar{\omega}_{i}=e^{-u} \omega_{i},
$$

and the structure equations (2.1) yield

$$
\tilde{\psi}^{*} \bar{\omega}_{i k}=\omega_{i k}+u_{k} \omega_{i}-u_{i} \omega_{k},
$$

where we expand $\pi^{*} \mathrm{~d} u=\sum_{k=0}^{n} u_{k} \omega_{k}$ for some smooth functions $u_{k}: F \rightarrow \mathbb{R}$. Note that $u_{0}$ is the $\nu$-pullback of the function $X$. Let $\bar{\tau}: \bar{U} \rightarrow M$ denote the $\bar{g}$-unit tangent bundle with canonical forms $\bar{\mu}, \bar{\omega}$ and $\psi: U \rightarrow \bar{U}$ the map which scales a $g$-unit vector by $e^{u}$. Then (2.8) implies that

$$
\psi^{*} \bar{\omega}=e^{-n u} \omega,
$$

and thus $\psi$ is a contact diffeomorphism. Moreover (2.8) and (2.9) yield

$$
\begin{aligned}
\psi^{*} \bar{\mu} & =-\frac{e^{-(n-1) u}}{n} \sum_{k=1}^{n}(-1)^{k-1}\left(\omega_{0 k}+u_{k} \theta-u_{0} \omega_{k}\right) \wedge \hat{\omega}_{k} \\
& =-e^{-(n-1) u}\left(\mu-X \omega+\frac{1}{n} \theta \wedge\left(i_{\nabla_{g} u} \omega\right)\right),
\end{aligned}
$$

which can be written as $\alpha \wedge \theta+\gamma \wedge(\mu-X \omega)$ for some $(n-1)$-form $\alpha$ and some smooth real-valued function $\gamma$ on $U$. This yields

$$
\psi^{*} \mathcal{M}_{e^{-2 u} g}=\mathcal{M}_{g, \nabla_{g} u} .
$$

Summarising we have proved

Proposition 2.1. Let $(M, g)$ be an oriented Riemannian manifold and $\mathbf{X}=\nabla_{g} u$ a gradient vector field. Then the $\mathbf{X}$-pseudosoliton M.A.S. on $(M, g)$ is equivalent to the minimal hypersurface M.A.S. on $\left(M, e^{-2 u} g\right)$. 
2.4. The nongradient case. Proposition 2.1 raises the question if there still exists a contact equivalence between minimal hypersurfaces and solitons if $\mathbf{X}$ is not a gradient vector field. We will argue next that this is not possible for $n \geq 2$, so assume in this subsection that $n \geq 2$. Before providing the arguments we recall a result from symplectic linear algebra. Suppose $(V, \Theta)$ is a symplectic vector space of dimension $2 n$, i.e. $\Theta \in \Lambda^{2}\left(V^{*}\right)$ is nondegenerate. If a form $\beta$ of degree $s \leq p$ satisfies

$$
\beta \wedge \Theta^{(n-p)}=0,
$$

then $\beta=0$. This is a corollary of the Lepage decomposition theorem for $p$-forms on symplectic vector spaces (cf. [10, Corollary 15.15]). Of course in our setting the symplectic vector spaces are the fibres of the contact subbundle $D$ and $\Theta$ is obtained by restricting $\mathrm{d} \theta$ to $D$.

Lemma 2.2. A necessary condition for the $\mathbf{X}$-pseudosoliton M.A.S. to be equivalent to the minimal hypersurface M.A.S. is the existence of an exact 1-form $\rho$ such that

$$
\mathrm{d}((\mu-X \omega) \wedge \theta)=\rho \wedge(\mu-X \omega) \wedge \theta .
$$

Proof. Write $\varphi=\mu-X \omega$ and suppose there exists a Riemannian metric $\bar{g}$ and a diffeomorphism $\psi: U \rightarrow \bar{U}$ such that $\psi^{*} \mathcal{M}_{\bar{g}}=\mathcal{M}_{g, \mathbf{X}}$. Then

$$
\psi^{*} \bar{\mu}=\alpha \wedge \theta+\beta \wedge \mathrm{d} \theta+\gamma \wedge \varphi,
$$

where $\alpha$ is an $(n-1)$-form, $\beta$ an $(n-2)$-form and $\gamma$ a smooth real-valued function on $U$. Note that we have

$$
\begin{aligned}
& 0=\varphi \wedge \mathrm{d} \theta, \\
& 0=\bar{\mu} \wedge \mathrm{d} \bar{\theta} .
\end{aligned}
$$

Wedging (2.12) with $\psi^{*} \mathrm{~d} \bar{\theta}$, using (2.13) and that $\psi$ is a contact diffeomorphism gives

$$
\left.(\beta \wedge \mathrm{d} \theta \wedge \mathrm{d} \theta)\right|_{D}=0,
$$

where $\left.\right|_{D}$ denotes the restriction to the contact subbundle $D \subset T U$. Now (2.14) together with (2.11) implies that $\left.\beta\right|_{D}=0$. This yields the existence of an $(n-1)$ form $\beta^{\prime}$ such that

$$
\beta=\beta^{\prime} \wedge \theta .
$$

We can therefore assume that there exists an $(n-1)$-form $\alpha^{\prime}$ such that

$$
\psi^{*} \bar{\mu}=\alpha^{\prime} \wedge \theta+\gamma \wedge \varphi .
$$

Wedging both sides of (2.15) with $\psi^{*} \bar{\theta}$ gives

$$
\psi^{*}(\bar{\mu} \wedge \bar{\theta})=\left(\alpha^{\prime} \wedge \theta+\gamma \wedge \varphi\right) \wedge \psi^{*} \bar{\theta} .
$$

This is equivalent to

$$
\psi^{*}(\bar{\mu} \wedge \bar{\theta})=\tilde{\gamma} \wedge \varphi \wedge \theta
$$

for some smooth nonvanishing real-valued function $\tilde{\gamma}$. Assume $\tilde{\gamma}$ is positive; otherwise just change signs in the following formulae. Since $\bar{\mu} \wedge \bar{\theta}$ is an exact form (see (2.3)), we must have

$$
\mathrm{d} \xi=\mathrm{d} f \wedge \xi
$$

where we have written $\xi=\varphi \wedge \theta$ and $f=-\ln |\tilde{\gamma}|$.

Using this lemma we can prove 
Theorem 2.3. The $\mathbf{X}$-pseudosoliton M.A.S. on an oriented Riemannian manifold $(M, g)$ of dimension $n+1 \geq 3$ is equivalent to a minimal hypersurface M.A.S. if and only if $\mathbf{X}$ is a gradient vector field.

Remark. Before giving the proof we point out identities which hold for the functions $X_{i}$ (recall (2.7) for their definition). Since $O=\left(\omega_{i k}\right) \in \mathcal{A}^{1}(F, \mathfrak{s o}(n+1))$ is a connection form we have $O\left(\mathbf{W}_{v}\right)=v$, where $\mathbf{W}_{v}$ is the vector field obtained by differentiating the flow

$$
\left(\left(p, e_{0}, \ldots, e_{n}\right), t\right) \mapsto\left(p, e_{0}, \ldots, e_{n}\right) \cdot \exp (t v)
$$

and $v \in \mathfrak{s o}(n+1)$, the Lie algebra of $S O(n+1)$. In particular this implies that the time $t$ flow of the vector field $\mathbf{W}_{i k}$ for $i<k$ maps the frame $\left(p, e_{0}, \ldots, e_{i}, \ldots, e_{k}, \ldots\right.$, $\left.e_{n}\right)$ to the frame

$$
\left(p, e_{0}, \ldots, \cos (t) e_{i}-\sin (t) e_{k}, \ldots, \sin (t) e_{i}+\cos (t) e_{k}, \ldots, e_{n}\right)
$$

and thus

$$
\mathcal{L}_{\mathbf{W}_{i k}} X_{j}=\delta_{j k} X_{i}-\delta_{i j} X_{k},
$$

where $\mathcal{L}$ stands for the Lie derivative.

Proof of Theorem 2.3. We have

$$
\mathrm{d} X_{0}=\sum_{i=0}^{n} P_{i} \omega_{i}-\sum_{k=1}^{n} X_{k} \omega_{0 k}
$$

for some smooth functions $P_{i}: F \rightarrow \mathbb{R}$. From this it follows with straightforward computations that the 1 -forms $\rho$ on $U$ which satisfy $\mathrm{d} \xi=\rho \wedge \xi$ pull back to $F$ to become

$$
\nu^{*} \rho=\lambda \omega_{0}+n \sum_{k=1}^{n} X_{k} \omega_{k}
$$

for a smooth function $\lambda: F \rightarrow \mathbb{R}$. Differentiating (2.17) gives

$$
\nu^{*} \mathrm{~d} \rho=\mathrm{d} \lambda \wedge \omega_{0}-\sum_{k=1}^{n} \omega_{0 k} \wedge \omega_{k}+n \sum_{k=1}^{n} \mathrm{~d} X_{k} \wedge \omega_{k}-n \sum_{i=0}^{n} \sum_{k=1}^{n} X_{k} \omega_{k i} \wedge \omega_{i} .
$$

Wedging with $\omega_{0} \wedge \hat{\omega}_{1}$ yields

$$
\nu^{*} \mathrm{~d} \rho \wedge \omega_{0} \wedge \hat{\omega}_{1}=\left(\lambda \omega_{01}-n \mathrm{~d} X_{1}-n \sum_{k=1}^{n} X_{k} \omega_{1 k}\right) \wedge \omega_{0} \wedge \omega .
$$

Using (2.16) we can expand

$$
\begin{aligned}
\mathrm{d} X_{1} \wedge \omega_{0} \wedge \omega & =\left(\left(\mathcal{L}_{\mathbf{W}_{01}} X_{1}\right) \omega_{01}+\sum_{k=1}^{n}\left(\mathcal{L}_{\mathbf{W}_{1 k}} X_{1}\right) \omega_{1 k}\right) \wedge \omega_{0} \wedge \omega \\
& =\left(X_{0} \omega_{01}-\sum_{k=1}^{n} X_{k} \omega_{1 k}\right) \wedge \omega_{0} \wedge \omega
\end{aligned}
$$

Concluding we get

$$
\nu^{*} \mathrm{~d} \rho \wedge \omega_{0} \wedge \hat{\omega}_{1}=\left(\lambda-n X_{0}\right) \omega_{01} \wedge \omega_{0} \wedge \omega .
$$


Suppose the $\mathbf{X}$-pseudosoliton M.A.S. on $(M, g)$ is equivalent to a minimal hypersurface M.A.S. Then, by Lemma 2.2 $\rho$ has to be exact. This implies that

$$
\lambda=n X_{0}
$$

and thus

$$
\nu^{*} \rho=n \sum_{i=0}^{n} X_{i} \omega_{i} .
$$

Note that if $\chi \in T F$ is a vector tangent to the frame $\left(p, e_{0}, \ldots, e_{n}\right)$, then we have

$$
\sum_{i=0}^{n}\left(X_{i} \omega_{i}\right)(\chi)=\sum_{i=0}^{n} g_{p}\left(g_{p}\left(\mathbf{X}(p), e_{i}\right) e_{i}, \pi^{\prime}(\chi)\right)=g_{p}\left(\mathbf{X}(p), \pi^{\prime}(\chi)\right)
$$

and thus

$$
\rho=n \tau^{*}\left(\mathbf{X}^{b}\right),
$$

where $\mathbf{X}^{b}$ denotes the $g$-dual 1 -form to $\mathbf{X}$. The 1 -form $\rho$ is exact and thus $\rho=\mathrm{d} f$ for some real-valued function $f$ on $U$ which is locally constant on the fibres of $\tau: U \rightarrow M$. Since the $\tau$-fibres are connected, it follows that $f$ is constant on the $\tau$-fibres and thus equals the pullback of a smooth function $u$ on $M$ for which

$$
d u=n \mathbf{X}^{b} .
$$

In other words $\mathbf{X}$ is a gradient vector field. Conversely if $\mathbf{X}$ is a gradient vector field, then the $\mathbf{X}$-pseudosoliton M.A.S. on $(M, g)$ is equivalent to the minimal hypersurface M.A.S. on $\left(M, e^{-2 u} g\right)$ by Proposition 2.1.

Remark. In [5], Bryant, Griffiths and Grossman study the calculus of variations on contact manifolds in the setting of differential systems. In particular they give necessary and sufficient conditions for an M.A.S. to be locally of Euler-Lagrange type, i.e. locally equivalent to an M.A.S. whose Legendre integral manifolds correspond to the solutions of a variational problem. In fact, if one replaces Lemma 2.2 with [5. Theorem 1.2], a proof along the lines of Theorem 2.3 shows that for $n \geq 2$ the $\mathbf{X}$-pseudosoliton M.A.S. is locally equivalent to an M.A.S. of Euler-Lagrange type if and only if $\mathbf{X}$ is a gradient vector field.

2.5. The surface case. Recall that in the case $n=1$ of a surface $(M, g)$, the solutions of the $\mathbf{X}$-pseudosoliton equation (1.1) are immersed curves on $M$ which may be reparametrised to become geodesics of a Weyl connection. In his Ph.D. thesis [12, the second author has constructed a 10-parameter family of Weyl connections on the 2-sphere whose geodesics are the great circles, and thus in particular projectively equivalent to the Levi-Civita connection of the standard spherical metric. Inspection shows that there are Weyl connections in this 10-parameter family whose vector field is not a gradient and thus they provide counterexamples to Theorem 2.3 in the surface case.

This raises the question of what are the necessary and sufficient conditions for the $\mathbf{X}$-pseudosolitons curves in order to be the geodesics of a Riemannian metric. In [12] it was also shown that locally on a surface every affine torsion-free connection is projectively equivalent to a Weyl connection. Finding the necessary and sufficient conditions thus comes down to finding the necessary and sufficient conditions for an affine torsion-free connection to be projectively equivalent to a Levi-Civita connection. Therefore the conditions follow by applying the results in [4, and we refer the reader to this source for further details. 


\section{ACKNOWLEDGEMENT}

The second author is grateful to Robert Bryant for helpful discussions.

\section{REFERENCES}

[1] Juan-Carlos Álvarez-Paiva and Gautier Berck, Finsler surfaces with prescribed geodesics, arXiv:math/1002.0243v1, 2010.

[2] Sigurd Angenent, On the formation of singularities in the curve shortening flow, J. Differential Geom. 33 (1991), no. 3, 601-633. MR.1100205

[3] Sigurd B. Angenent, Shrinking doughnuts, Nonlinear diffusion equations and their equilibrium states, 3 (Gregynog, 1989), Progr. Nonlinear Differential Equations Appl., vol. 7, Birkhäuser Boston, Boston, MA, 1992, pp. 21-38. MR.1167827

[4] Robert Bryant, Maciej Dunajski, and Michael Eastwood, Metrisability of two-dimensional projective structures, J. Differential Geom. 83 (2009), no. 3, 465-499. MR2581355

[5] Robert Bryant, Phillip Griffiths, and Daniel Grossman, Exterior differential systems and Euler-Lagrange partial differential equations, Chicago Lectures in Mathematics, University of Chicago Press, Chicago, IL, 2003. MR1985469

[6] Julie Clutterbuck, Oliver C. Schnürer, and Felix Schulze, Stability of translating solutions to mean curvature flow, Calc. Var. Partial Differential Equations 29 (2007), no. 3, 281-293. MR2321890

[7] Gerhard Huisken, Asymptotic behavior for singularities of the mean curvature flow, J. Differential Geom. 31 (1990), no. 1, 285-299. MR.1030675

[8] N. Hungerbühler and K. Smoczyk, Soliton solutions for the mean curvature flow, Differential Integral Equations 13 (2000), no. 10-12, 1321-1345. MR1787070

[9] Norbert Hungerbühler and Beatrice Roost, Mean curvature flow solitons, Analytic aspects of problems in Riemannian geometry: Elliptic PDEs, solitons and computer imaging, Séminaires et Congrès, vol. 19, Société Mathématiqe de France, 2009, pp. 129-158.

[10] Paulette Libermann and Charles-Michel Marle, Symplectic geometry and analytical mechanics, Mathematics and its Applications, vol. 35, D. Reidel Publishing Co., Dordrecht, 1987. Translated from the French by Bertram Eugene Schwarzbach. MR 882548

[11] V. V. Lychagin, V. N. Rubtsov, and I. V. Chekalov, A classification of Monge-Ampère equations, Ann. Sci. École Norm. Sup. (4) 26 (1993), no. 3, 281-308. MR1222276

[12] Thomas Mettler, On the Weyl metrisability problem for projective surfaces and related topics, Ph.D. thesis, Université de Fribourg, 2010.

[13] Knut Smoczyk, A relation between mean curvature flow solitons and minimal submanifolds, Math. Nachr. 229 (2001), 175-186. MR.1855161

Department of Mathematics, ETH Zürich, CH-8092 Zurich, Switzerland

E-mail address: norbert.hungerbuehler@math.ethz.ch

Department of Mathematics, University of California at Berkeley, Berkeley, CaliFORNIA 94720

E-mail address: mettler@math.berkeley.edu 